\title{
Images of the month 1: 'Soy sauce' pleural effusion: what causes black pleural fluid?
}

\author{
Authors: Joseph Newman, ${ }^{A}$ Tejas Ingle, ${ }^{A}$ She Lok $^{\mathrm{B}}$ and Liana Pradan ${ }^{\mathrm{B}}$
}

We present a case of black pleural fluid following thoracic trauma. The unusual dark colour most strikingly resembled soy sauce as independently commented upon by multiple treating physicians. The black colouration could not be fully accounted for by haemothorax or cholethorax, so other differential diagnoses were investigated, including Aspergillus niger infection and malignant melanoma. The cause, however, was thought to be due to staining of the fluid with carbon deposited in the pleural space from the non-volatilised impurities from smoking crack cocaine. A novel use of a point-of-care urine toxicology assay confirmed the presence of cocaine in the pleural fluid. Considering a broad range of differential diagnoses is needed to avoid missing important causes of unusual pleural effusions.

KEYWORDS: respiratory medicine, black pleural fluid, pleural effusion, crack cocaine

DOI: 10.7861/clinmed.2021-0403

\section{Case presentation}

A 34-year-old man presented to the emergency department with a single penetrating stab wound to the anterolateral aspect of the right hemithorax. The patient regularly injected heroin intravenously and smoked both crack cocaine and cannabis.

A surgical chest drain was inserted. $1.8 \mathrm{~L}$ of black non-viscous pleural fluid (Fig 1) was drained within 48 hours. Multiple colleagues independently commented that it closely resembled soy sauce in colour and consistency.

Computed tomography showed a penetrating right lung laceration with hydropneumothorax, fifth rib fracture anteriorly, surgical emphysema and an appropriately sited chest drain (Fig 2). There was a possible but radiologically inconclusive liver laceration, but no oesophageal injury.

Blood tests showed a stable haemoglobin of $116 \mathrm{~g} / \mathrm{L}$, excluding significant haemorrhage.

Biochemical analysis of the pleural fluid showed $\mathrm{pH} 7.8$, protein of $27 \mathrm{~g} / \mathrm{L}$, amylase $<1 \mathrm{U} / \mathrm{L}$ and bilirubin of $345 \mu \mathrm{mol} / \mathrm{L}$ (relative

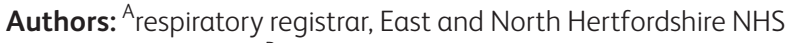
Trust, Stevenage, UK; ${ }^{B}$ respiratory consultant, East and North Hertfordshire NHS Trust, Stevenage, UK to serum value of $7 \mu \mathrm{mol} / \mathrm{L}$ ) suggestive of a bile leak. ${ }^{1}$ Cytology showed no malignant cells. No bacteria or fungi were cultured. Aspergillus serology was negative.

\section{Discussion}

The 'soy sauce' colour of the fluid was not fully explained, however, by the red pigmentation of a haemothorax or the light green of a cholethorax. There are no known reports in the literature of fresh blood or bile, nor both together, causing black colouration of pleural fluid. Blood left in the pleural cavity over time can

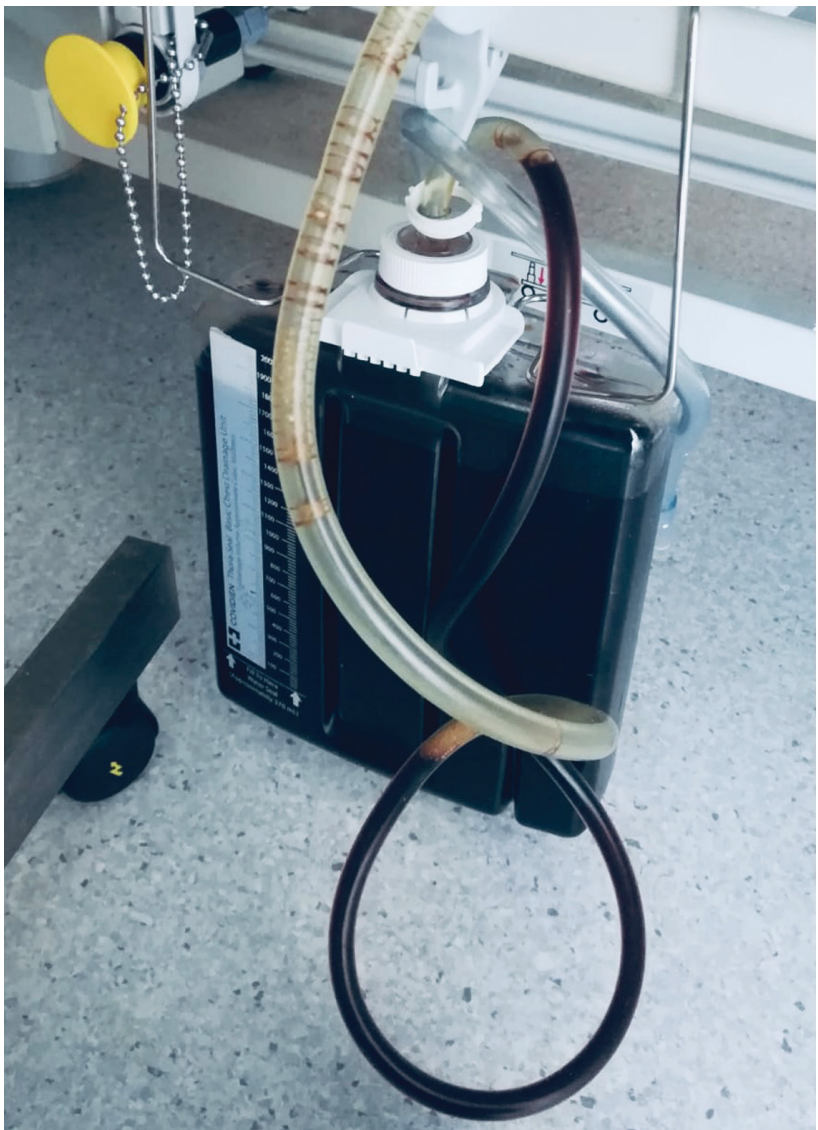

Fig 1. A large volume of 'soy sauce' black coloured pleural fluid drained from the chest. 
Fig 2. Axial computed tomography of the thorax. a) Penetrating injury (black arrow) with rib fracture, surgical emphysema and lung laceration. b) Hydropneumothorax with surgical emphysema and appropriately sited chest drain.

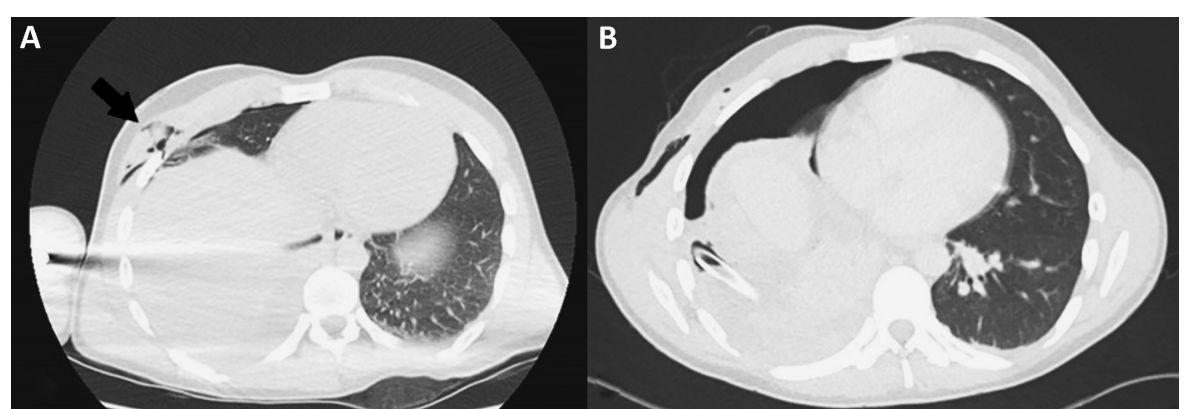

haemolyse and turn darker in colour, but this effusion was drained within an hour of the trauma occurring. ${ }^{2}$

Black pleural effusions are extremely rare with scant case reports. The differential diagnosis includes malignant melanoma, pancreatic pseudocyst and infection with Aspergillus niger. ${ }^{3}$ (Incidentally, various species of Aspergillus are used in the fermentation process in the production of soy sauce, notably

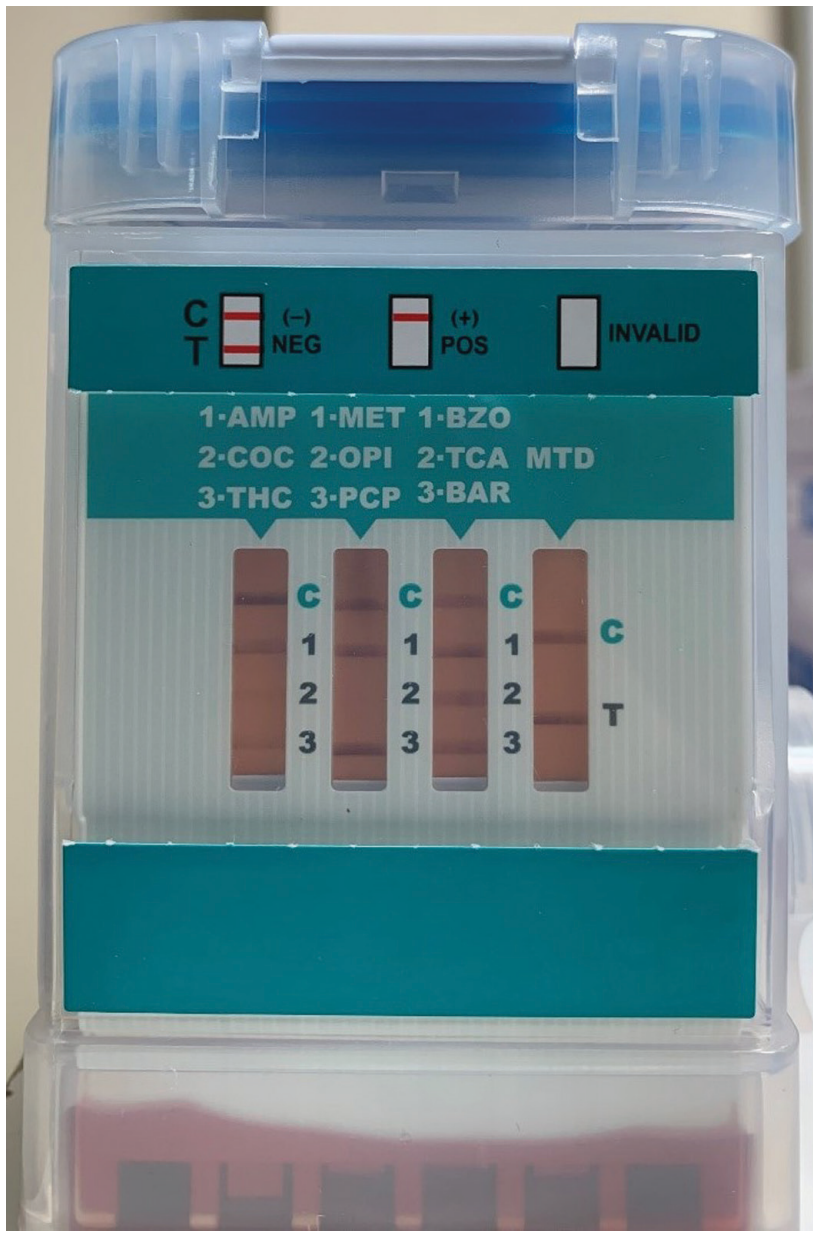

Fig 3. Pleural fluid then tested directly with a urine toxicology point-ofcare assay. A positive test on this toxicology assay is shown by the absence of a line next to the corresponding drug relative to the control, in this case, confirming the presence of cocaine (COC), cannabis (THC) and opiates (OPI).
A oryzae.) An unremarkable dermatological examination with the earlier investigations excluded these causes, leaving the black colouration unexplained.

Given the patient's recreational drug use, the pleural fluid was then tested directly with a urine toxicology point-of-care assay (Fig 3). This confirmed the presence of cocaine, cannabis and opiates. As per correspondence with the manufacturer, this is a novel use of this investigation. While not validated, it does suggest direct pulmonary exposure to crack cocaine. Had the drug abuse not been apparent from the history, then this confirmatory test would be all the more important here.

It has been shown cytologically that macrophages in the pleural fluid of crack smokers become laden with carbon from the non-volatilised impurities. ${ }^{4}$ The carbon transfers from the parenchyma to the visceral pleura when clearance mechanisms are overwhelmed. ${ }^{4}$ Smoking crack cocaine is rarely a primary cause of pleural effusion, but if fluid accumulates for any other reason, it can be stained black by deposition of carbon pigments.

While we have referred to this crack cocaine-related black pleural fluid as similar to 'soy sauce', please note that this should not be confused with the classic 'anchovy sauce' of amoebic pleural infections.

When the colour of pleural fluid is unusual, unexpected or not easily accounted for, a broad differential diagnosis should be considered and further investigations undertaken so that important causes are not missed..

\section{References}

1 Darwin P, Goldberg E, Uradomo L. Jackson Pratt drain fluid-toserum bilirubin concentration ratio for the diagnosis of bile leaks. Gastrointestinal Endoscopy 2010;71:99-104.

2 Jayakrishnan B, Dildar B, Rizavi DM, Mubaihsi SMA, Kindi AA. Black pleural effusion. Lancet 2015;386:e7.

3 Saraya T, Light RW, Takizawa H, Goto H. Black pleural effusion. Am J Med 2013;126:641.

4 Singh B, Greenebaum E, Cole R. Carbon-laden macrophages in pleural fluid of crack smokers. Diagnostic Cytopathology 1995;13:316-9.

Address for correspondence: Dr Joseph Newman, Department of Respiratory Medicine, Lister Hospital, Coreys Mill Lane, Stevenage, Hertfordshire SG1 4AB, UK. Email: joseph.newman@nhs.net 